Second Meeting, 12 th December, 1902.

Dr ThIad in the Chair.

Mathematical Correspondence.

Robert Smgox, Matthew Stewart, Jamks Stirling.

[See page 2.]

\title{
On the Uniqueness of Solution of the Linear Differential Equation of the Second Order.
}

By Dr Peddie.

1. In many problems of physics, even in widely different branches of the subject, the relation satisfied by the variables is expressible by means of a linear differential equation of the second order. In general, "initial" conditions have also to be satisfied. If the equation truly represents the physical conditions in, for example, some case of motion, and if no state of instability exists, the solution must be unique. But it is impossible in any case to say with absolute certainty that the representation is strict. The possible error depends on the error which may be made in observation or experiment, and on the number of independent observations or experiments the results of which have been used as the basis of the "law" expressed by the equation. The probable accuracy of any statement as to the non-existence of instability is also dependent on the rigour and extent of the observational or experimental groundwork. The physicist therefore frequently assumes a form of solution which suits his conditions, and does not trouble himself to enquire whether or not other solutions exist if he finds that the one which he has obtained corresponds sufficiently closely to his facts. This procedure is thoroughly justifiable, seeing that he is as sure of the accuracy of his result as he is of the accuracy of his 
original equation, while on the other hand a proof of uniqueness may not be easy to obtain. Even if other solutions were found to exist he would be justified in retaining his own and asserting that constraints, whose action did not appear in the differential equation, prevented the manifestation of instability and so prevented the applicability of the other solutions. At least he would be so justified except in the event of such a solution suiting his facts better than his own did. Yet the fact that such a better solution may be found makes the farther investigation of the question desirable, and the proof of the existence of uniqueness adds, when the accuracy of the solution is verified by experiment, to the probability of the accuracy of the differential equation as a description of facts.

2. A well-known example in which uniqueness of solution is proved is furnished by Laplace's Equation in the theory of potential. The proof is obtained by an application of Green's Theorem. Another example occurs in the theory of the conduction of heat (Thomson and Tait's Treatise on Natural Philosophy), Green's Theorem being extended so as to apply.

A similar use of Green's Theorem is made by Picard in his Traite d'Analyse. He proves that the solution of the equation

$$
a \frac{\partial^{2} u}{\partial x^{2}}+2 b \frac{\partial^{2} u}{\partial x \partial y}+c \frac{\partial^{2} u}{\partial y^{2}}+2 d \frac{\partial u}{\partial x}+2 e \frac{\partial u}{\partial y}+f u=0
$$

is unique if $b^{\prime}-a c$ be negative while $u$ has a given succession of values along a sufficiently small closed contour in the $x, y$ plane. The coefficients are any continuous functions of $x$ and $y$, and $a$ and $c$ are alike in sign.

In the case in which $f$ has a sign opposite to that of $a$ and $c$, the limitation as to smallness of the contour is not required, but the proof is based on the assumption that the coefficients are analytic functions.

Paraf (Ann. de la Faculté des Sciences de Toulouse, 1892) gives a proof of the uniqueness of the solution when $a c>b^{2}$, apart from any assumption as to the nature of the quantities, when $f$ is zero or of opposite sign to $a$ and $c$.

The object of the present investigation is to obtain a criterion by means of which we can investigate the problem when $f$ is not of opposite sign to $a$ and $c$. That restriction, however, is replaced by another. 


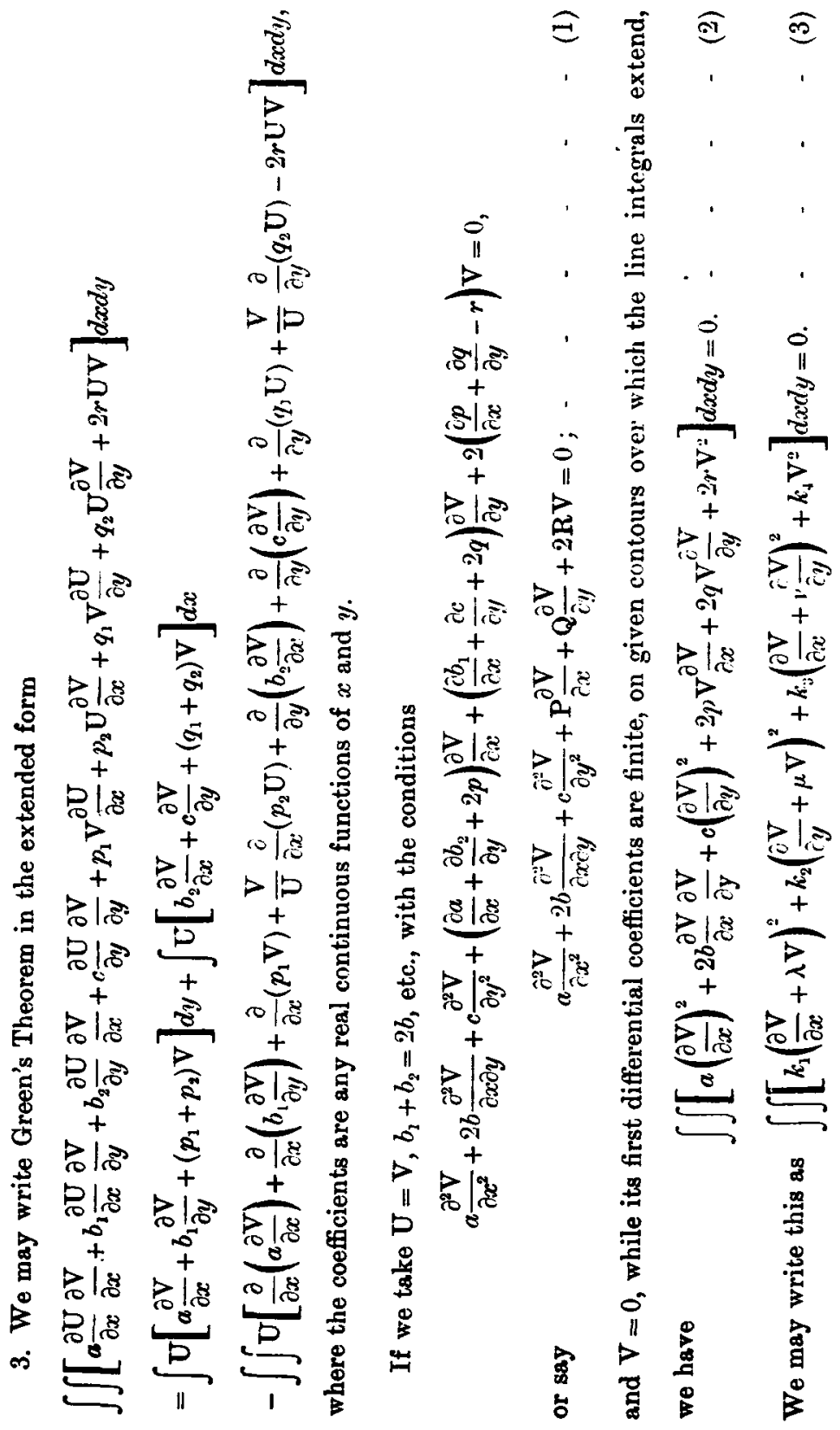


Equating coefficients we get

$$
\begin{array}{ll}
a=k_{1}+k_{3}, & p=\lambda k_{1}, \\
b=\nu k_{3}, & 2 r=\frac{p^{2}}{k_{1}}+\frac{q^{2}}{k_{3}}+k_{4} . \\
c=k_{3}+\nu^{2} k_{3}, & q=\mu k_{2},
\end{array}
$$

If the $\mathrm{ks}$ be all of one sign, (3) can only be satisfied by

$$
\mathrm{V}=0, \frac{\partial \mathrm{V}}{\partial x}=0, \frac{\partial \mathrm{V}}{\partial y}=0 \text {. }
$$

Therefore, since $V$ may be supposed to be the difference of two functions each of which is assumed, if possible, to satisfy (1), there cannot be more than one solution of the linear equation (1).subject to the condition that the function shall have a given succession of values along given contours.

4. The condition that the $k$ s shall be of one sign necessitates $a$ and $c$ being of one sign, which we may consider to be positive along with those of the $k \mathrm{~s}$. And the relations among the coefficients give

$$
b^{2}=\left(a-k_{1}\right)\left(c-k_{2}\right),
$$

while $a \nless k_{1}, c \nless k_{2}$, so that, as in Picard's cases, we have

$$
a c-b^{2} \nless 0 \text {. }
$$

5. From the conditions

we find

$$
\begin{aligned}
& \frac{\partial a}{\partial x}+2 \frac{\partial b}{\partial y}-\frac{\partial b_{1}}{\partial y}+2 p=\mathbf{P} \\
& \frac{\partial b_{1}}{\partial x}+\frac{\partial c}{\partial y}+2 q=\mathrm{Q} \\
& \frac{\partial p}{\partial x}+\frac{\partial q}{\partial y}-r \quad=\mathrm{R}
\end{aligned}
$$

$$
2 r=\frac{\partial \mathrm{P}}{\partial x}+\frac{\partial \mathrm{Q}}{\partial y}-\frac{\partial^{2} a}{\partial x^{2}}-2 \frac{\partial^{2} b}{\partial x \partial y}-\frac{\partial^{2} c}{\partial y^{2}}-2 \mathrm{R},
$$

so that the value of $r$ is known, and therefore that of

is known.

$$
2 r=\frac{p^{2}}{a-k_{3}}+\frac{q^{2} k_{3}}{c k_{3}-b^{2}}+k_{4}
$$


The quantity $r$ must essentially be positive, and

$$
k_{3}<a>\frac{b^{2}}{c} \text {. }
$$

We see therefore that the solution of

$$
a \frac{\partial^{2} \mathrm{~V}}{\partial x^{2}}+2 b \frac{\partial^{2} \mathrm{~V}}{\partial x \partial y}+c \frac{\partial^{2} \mathrm{~V}}{\partial y^{2}}+\mathrm{P} \frac{\partial \mathrm{V}}{\partial x}+\mathrm{Q} \frac{\partial \mathrm{V}}{\partial y}+2 \mathrm{RV}=0
$$

is unique if the contours upon which $\mathrm{V}$ is given do not extend to the negative sides of the curves

$$
\begin{gathered}
a c-b^{2}=0, \\
2 r=\frac{\partial \mathrm{P}}{\partial x}+\frac{\partial \mathrm{Q}}{\partial y}-\frac{\partial^{2} a}{\partial x^{2}}-2 \frac{\partial^{2} b}{\partial x \partial y}-\frac{\partial^{2} c}{\partial y^{2}}-2 \mathrm{R}=0, \\
2 r-\frac{\left[\mathrm{P}-\frac{\partial a}{\partial x}-2 \frac{\partial b}{\partial y}+\frac{\partial b_{1}}{\partial y}\right]^{2}}{4\left(a-k_{3}\right)}-\frac{k_{3}\left[\mathrm{Q}-\frac{\partial c}{\partial y}-\frac{\partial b_{1}}{\partial x}\right]^{2}}{4\left(c k_{3}-b^{2}\right)}=0 .
\end{gathered}
$$

6. The two latter conditions become identical when

$$
2(r+\mathrm{R})=\frac{\partial \mathrm{P}}{\partial x}+\frac{\partial \mathbf{Q}}{\partial y}-\frac{\partial^{2} a}{\partial x^{2}}-2 \frac{\partial^{2} b}{\partial x \partial y}-\frac{\partial^{2} c}{\partial y^{2}}=0,
$$

and the condition then is that $R$ shall be negative-a special case of Paraf's result.

If the first two conditions are satisfied, the problem reduces to that of the possibility of determining $b_{1}$ and $k_{3}$ so 23 to satisfy the third. The extreme cases occur when $k_{3}=a$ and $k_{3} c=b^{2}$. If $k_{3}=a$ we have $k_{1}=0, p=0$, and the third condition becomes

$$
a\left[\int(\mathrm{R}+r) d y+f(x)\right]^{2} \ngtr 2 r\left(a c-b^{2}\right) .
$$

If $k_{3} c=b^{2}$ it becomes

$$
c\left[\int(\mathrm{R}+r) d x+\phi(y)\right]^{2} \ngtr 2 r\left(a c-b^{2}\right) .
$$

The solution is unique when $f(x)$ or $\phi(y)$ can be chosen so as to suit the inequality.

The curve (6) is a boundary flexible within limits, such that, if it be capable of deformation so as to have the given contours entirely 6 Vol. 21 
on its positive side, the solution is unique. In determining whether or not sufficient deformation be possible it might be convenient to assign to $q$ an arbitrary value and use (6) in the form

$$
2 r-\frac{\left[\mathbf{P}-\frac{\partial a}{\partial x}-2 \frac{\partial b}{\partial y}+\psi(y)+\int \frac{\partial}{\partial y}\left(\mathbf{Q}-\frac{\partial c}{\partial y}-2 q\right) d x\right]^{2}}{4\left(a-b_{3}\right)}-\frac{k_{3} q^{2}}{4\left(c k_{3}-b^{2}\right)}=0
$$

where $\psi$ is arbitrary. But we shall find that it is possible to dispense with (6).

7. It is possible to explore the $x, y$ plane further, even where the value of $r$ is negative. For this purpose an artifice, similarly employed by Picard, may be used. He remarks that, if $B$ and $B^{\prime}$ be any two continuous functions of $x$ and $y$, we have

$$
\iint\left[\frac{\partial\left(\mathbf{B V}^{2}\right)}{\partial x}+\frac{\partial\left(\mathbf{B ~ V}^{2}\right)}{\partial y}\right] d x d y=0
$$

since $\mathrm{V}$ is zero on the contours when we suppose it to represent the difference of two functions satisfying (1). Thus (2) can be written

$$
\begin{gathered}
\iint\left[a\left(\frac{\partial \mathrm{V}}{\partial x}+\frac{\mathrm{B}}{a} \mathrm{~V}\right)^{2}+2 b \frac{\partial \mathrm{V}}{\partial x} \frac{\partial \mathrm{V}}{\partial y}+c\left(\frac{\partial \mathrm{V}}{\partial y}+\frac{\mathrm{B}^{\prime}}{c} \mathrm{~V}\right)^{2}+2 p \mathrm{~V} \frac{\partial \mathrm{V}}{\partial x}+2 q \mathrm{~V} \frac{\partial \mathrm{V}}{\partial y}\right. \\
\left.+\left(2 v+\frac{\partial \mathrm{B}}{\partial x}+\frac{\partial \mathrm{B}}{\partial y}-\frac{\mathrm{B}^{\prime}}{a}-\frac{\mathrm{B}^{\prime}}{c}\right)^{2} \mathrm{~V}^{2}\right] d x d y=0 .
\end{gathered}
$$

Equating coefficients we have

$a=k_{1}+k_{3}$,

$b=v k_{3}$, $c=k_{2}+v^{2} k_{3}$

$$
\begin{aligned}
& p+\mathrm{B}=\lambda k_{1}, \\
& q+\mathrm{B}^{\prime}=\mu k_{2},
\end{aligned}, \quad 2 r+\frac{\partial \mathrm{B}}{\partial x}+\frac{\partial \mathrm{B}^{\prime}}{\partial y}=\frac{(p+\mathrm{B})^{2}}{k_{1}}+\frac{\left(q+\mathrm{B}^{\prime}\right)^{2}}{k_{2}}+k_{1} .
$$

We may assume $\mathrm{B}=-p, \mathrm{~B}^{\prime}=-q$, and hence we find that when the contours on which $V$ is given do not lie, in whole or in part, on the negative side of the curve

$$
2(r-\mathbf{R})=\frac{\partial \mathbf{P}}{\partial x}+\frac{\partial Q}{\partial y}-\frac{\partial^{2} a}{\partial x^{2}}-2 \frac{\partial^{2} b}{\partial x \partial y}-\frac{\partial^{2} c}{\partial y^{2}}-4 \mathbf{R}=0,
$$

the solution is unique. Thus, provided that the value of $\mathbf{R}$ do not exceed that of $r$, we know that the solution is unique even when $R$ is positive. 
If we choose $p=0, \mathrm{~B}^{\prime}=-q$, we get

$$
\frac{\partial \mathrm{B}}{\partial x}=\frac{\mathbf{B}^{2}}{k_{1}}+k_{i}+\mathbf{R}-r
$$

and can consider the case where $r-\mathrm{R}$ is negative. Jet $-\mathrm{A}^{2} / k_{1}$ be its greatest negative value, and take $k_{4}=\mathrm{A}^{2} / k_{1}-(\mathrm{R}-r), k_{1}$ being assumed constant. These conditions give

$$
\mathbf{B}=\mathbf{A} \tan \frac{\mathbf{A}}{k_{1}}(x+\alpha) \text {. }
$$

By suitable choice of $a$, we have $B$ remaining continuous throughout any strip of the $x, y$ plane, parallel to the $y$ axis, whose breadth is less than $\pi k_{1} / \mathrm{A}$; that is, less than

$$
\delta=\frac{\pi\left(a_{0} c_{0}-b_{0}{ }^{2}\right)}{c_{0} \mathrm{~A}},
$$

where the least value of $\frac{a c-l^{2}}{c}$ is taken, and $\frac{2 \mathrm{~A}^{\prime \prime} c_{0}}{a_{0} c_{0}-b_{0}{ }^{2}}$ is the greatest positive value of

$$
\frac{\partial^{2} a}{\partial x^{2}}+2 \frac{\partial^{2} b}{\partial x \partial y}+\frac{\partial^{2} c}{\partial y^{2}}-\frac{\partial \mathbf{P}}{\partial x}-\frac{\partial \mathbf{Q}}{\partial y}+4 \mathbf{R}
$$

If $a_{0}<c_{0}$ we would take $q=0, \mathrm{~B}=-p$ and so get $a_{0}$ instead of $c_{0}$ in the denominator of $\delta$.

If we refer to a new set of rectangular axes, the new $x$-axis being selected parallel to the least breadth of a given closed contour on which $\mathrm{V}$ has a given succession of values, we can determine whether or not we can assert that uniqueness of solution exists under the given condition.

Since $\delta$ is never zero, the solution of (1) is always unique, as Picard shows, when the contour is sufficiently small.

Another test is given by taking $B=p=0, B^{\prime}=[\psi(x)-1] q$. We then get

$$
(r-\mathrm{R})+(r+\mathrm{R}) \psi(x)=\frac{\left[\psi(x) \int(\mathrm{R}+r) d y\right]^{2}}{k_{2}}+k_{4} .
$$

Otherwise we may take $\mathrm{B}^{\prime}=q=0, \mathrm{~B}=[\phi(y)-1] p$, which gives

$$
(r-\mathbf{R})+(r+\mathbf{R}) \phi(y)=\frac{\left[\phi(y) \int(\mathbf{R}+r) d x\right]^{2}}{k_{2}}+k_{4} .
$$

If the disposable quantities can be chosen to satisfy either condition, the solution is unique. 


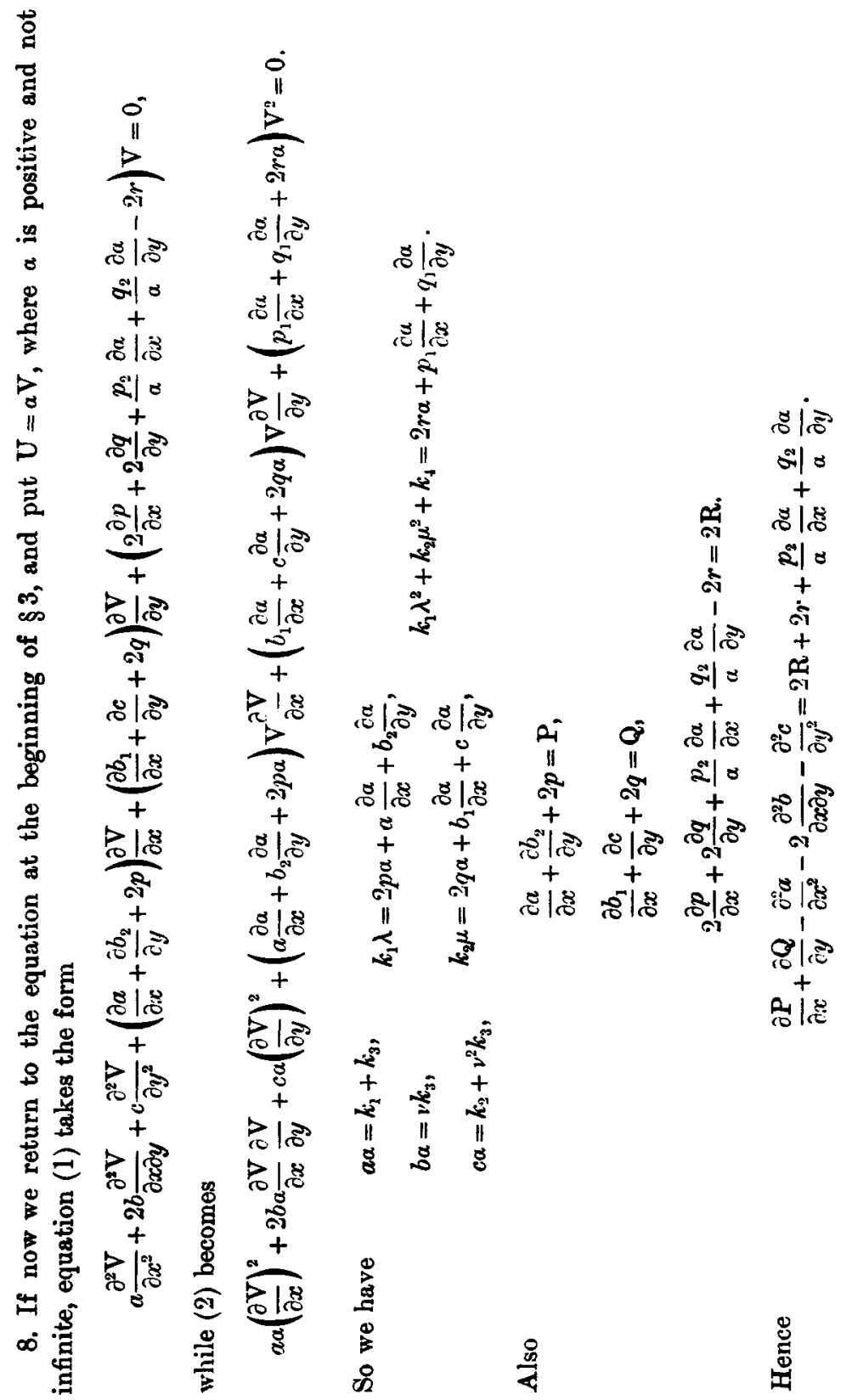



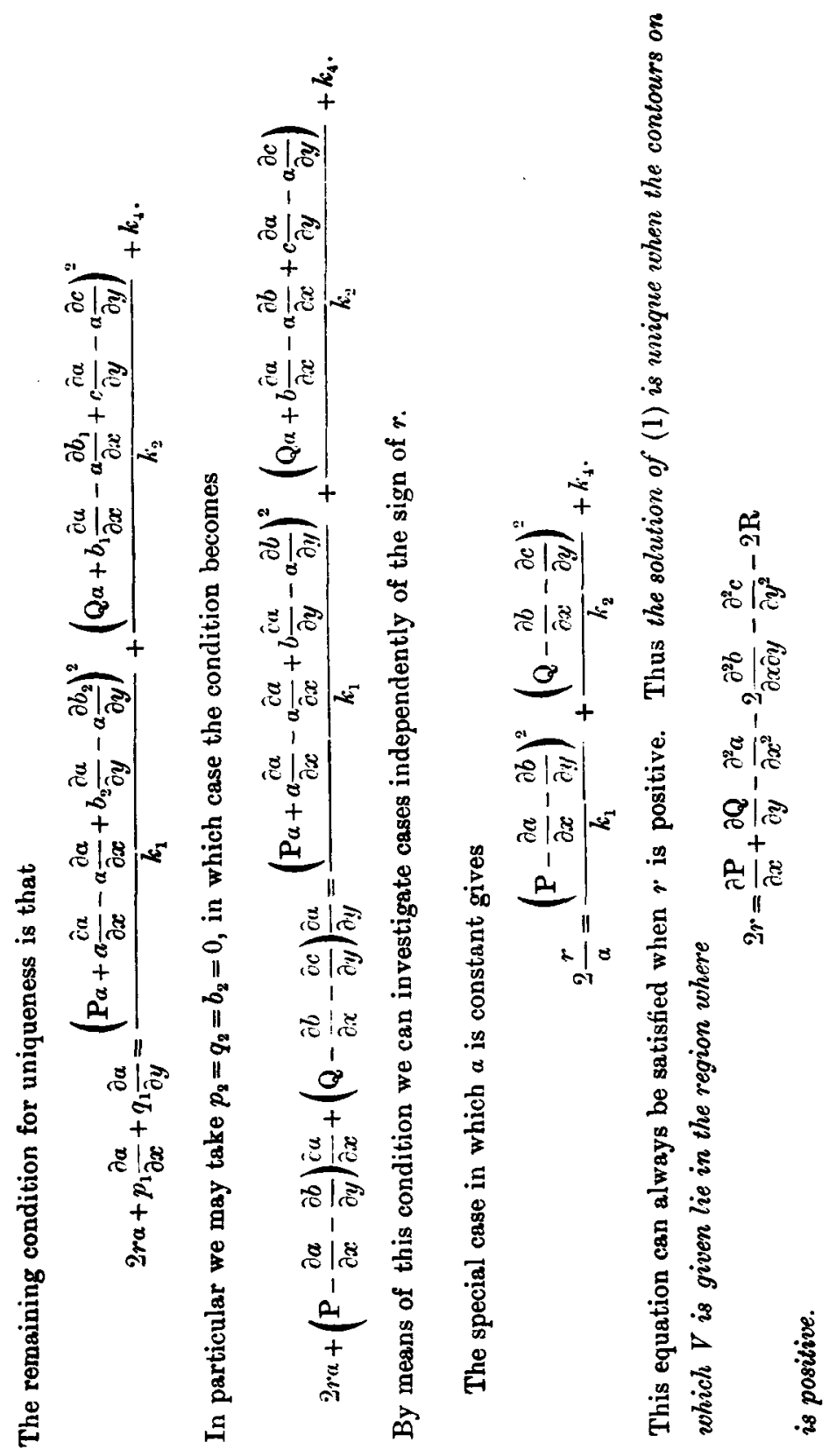
9. We may extend the investigation to regions where $r$ is negative hy means of the method used in $\$ 7, \alpha$ being constant. The equation becomes

$$
2 r a+\frac{\partial \mathrm{B}}{\partial x}+\frac{\partial \mathrm{B}^{\prime}}{\partial y}=\frac{(p+\mathrm{B})^{2}}{k_{1}}+\frac{\left(q+\mathrm{B}^{\prime}\right)}{k_{y}}+k_{4} .
$$

Putting $p=0, \mathrm{~B}^{\prime}=-q$, we find

$$
\frac{\hat{n} \mathbf{B}}{i_{i}}=\frac{\mathbf{b}^{2}}{k_{1}}+k_{+}-(\mathrm{R}+r)+2 r a \text {. }
$$

Taking $k_{4}=\mathbf{A}^{\prime \prime} / k_{1}-(\mathrm{R}+r)+2 r a$, where $\mathrm{A}^{\prime \prime} / k_{1}$ is the greatest positive value of $\mathrm{R}+r(1-2 \alpha), k_{1}$ being constant, we see that $\mathrm{B}$ can be made continuous throughout any strip, parallel to the $y$ axis, whose breadth does not exceed

$$
\pi \frac{k_{i}}{\mathrm{~A}} \text {. }
$$

When $R$ is positive and $r$ negative, the value of $\alpha$ which makes this quantity a maximum should be chosen. As the least value of $k_{3}$ is $b^{\prime \prime} / c a$, the greatest value of $k_{1}$ is $\left(a c \alpha^{\prime \prime}-b^{\prime \prime}\right) / c \alpha$. The largest constant value which $k_{1}$ can be given cannot exceed the smallest value which $\left(a c a^{2}-b^{2}\right) / c u$ can take when $a$ is tixed.

By putting

$$
\mathbf{B}=p=0, \mathbf{B}^{\prime}=(\psi(x)-1) q, \text { or } \mathbf{B}^{\prime}=q=0, \mathbf{B}=(\psi(y)-1) p,
$$

respectively, we get

$$
\begin{array}{r}
2 r a+(\psi(x)-1)(\mathrm{R}+r)=\frac{\left[\psi(x) \int(\mathrm{R}+r) d y\right]^{2}}{k_{2}}+k_{4}, \\
\text { or } \quad 2 r a+(\psi(y)-1)(\mathrm{R}+r)=\frac{\left[\psi(y) \int(\mathrm{R}+r) d x\right]^{2}}{k_{2}}+k_{4},
\end{array}
$$

instead of the conditions given at the end of $\$ 7$, the positive con. stant $a$ being now at disposal in addition to the other quantities.

If we put $\mathrm{B}=-p, \mathrm{~B}^{\prime}=-q$, the condition, with $a$ constant, becomes

$$
2 r(a-1)-\mathbf{R}=k_{4}
$$


Hence we see that, even if $R$ be positive and $r$ be negative, the solution is unique provided that $2 r(a-1)>R, a<1$.

10. There is no limitation on the size of the contours over which $\mathrm{V}$ is given, where $r$ and $\mathrm{R}$ have the same sign.

By change of the variables an equation may often be put into a form in which one or other of the conditions $R$ negative, or $r$ positive, holds.

The methods above used may be extended to the case of more than two independent variables. 